Technical Memorandum 86067

\title{
Detection of Bump-on-Tail Reduced Electron Velocity Distributions at the Electron Foreshock Boundary
}

R.J. Fitzenreiter, A.J. Klimas and J.D. Scudder

FEBRUARY 1984

National Aeronautics and

Soace Administration

Goddard Space Flight Center

Greenoelt. Maryland 20 $\bar{i} i$ 
DETECTION OF BUMP-ON-TAIL REDUCED ELECTRON VELOCITY DISTRIBUTIONS AT THE ELECTRON FORESHOCK BOUNDARY

\author{
Richard J. Fitzenreiter \\ Alexander J. KI imas \\ and \\ Jack D. Scudder \\ NASA/Goddard Space Flight Center \\ Laboratory for Extraterrestrial Physics \\ Greenbelt, MD 20771
}

ACCE PTED BY: Geophysical Research Letters 
Reduced velocity distributions are derived from three-dimensional measurements of the velocity distribution of electrons in the 7-500 eV range in the electron foreshock. Bump-on-tail reduced distributions are presented for the first time at the foreshock boundary consistent with filbert and Kellogg's proposed time-of-flight mechanism for generating the electron beans. In a significant number of boundary crossings, bump-on-tall reduced distributions were found in consecutive $3 \mathrm{sec}$ measurements made $9 \mathrm{sec}$ apart. We conclude that, although the beams are linearly unstable to plasma waves according to the Penrose criterion, they persist on a time scale of 3-15 sec. 
Introduction

Upstream from the earth's bow shock, enhanced fluxes of energetic electrons are observed when the local magnetic fleld connects to the shock surface lOgilvie et al., 1971; Feldman et al., 1973; Anderson et al., 1979; Feldman et al., 1982]. This is the electron component of the earth's foreshock [Greenstadt, 1976] and occupies the space behind the surface of tangent magnetic field lines as shown in Figure 1 . Closely associated wh the enhanced electron $\mathrm{fluxes}$ is electrostatic plasma noise that is most intense at the upstream edge of the foreshock [Scarf et al., 1971; Filbert and Kellogg, 1979; Anderson et al., 1981]. Filbert and Kellogg [1979] proposed that the plasma noise could be generated by the bump-on-tail class of reduced electron velocity distributions, although at the time, such velocity distributions had not yet been observed. The basis for this proposal was that the upstream edge of the foreshock is spatially dispersed with respect to parallel velocity due to the solar wind electric field drift of the particles. Electrons originating on connecting field lines at the bow shock with the highest parallel velocity move upstream along a resultant path that is closest to the tangent field line, while electrons with lower parallel velocity cross-field drift for a longer time and are carried further downstream (see Figure 1). This time-of-flight separation produces a spatial variation with respect to parallel velocity and was suggested by filbert and Kellogg as the mechanism for the existence of peaked reduced velocity distributions at the foreshock boundary. Recently, Fung et al. [1982] have suggested that the foreshock plasma noise can be produced by electron velocity lisr,ibutions which are not of the bump-on-tall class postulated by Fllbert and Kellogg. The motivation for the distribution suggested by Fung et al. was the lack of observational evidence for the bump-on-tall. In this letter, we present new experimental findings which bear on these theoretical discussions.

Recent electron plasma measurements confirm the velocity dispersive boundary model; $1 . e .$, when the magnetzc field configuration changes in such a way that the foreshock boundary is swept past the observing spacecraft, the energy at which the onset of upstream electrons occurs is found to decrase with increasing penetration Into the foreshock [Anderson et al., 1979; Feldman et al., 1983 ]. Peaks in fast ( 3 second) two-dimensional measurements at the 
foreshock boundary have been reported by Feldman et al. [1983], and peaks in energy spectra of electrons moving upstream from the bowshock have been measured by Gurnett and Frank [1975]. These measurements, however, do not address the presence of mult1-peaked reduced distributions as postulated by Filbert and Kellogg. It is the purpose of this paper to present the required fast, three-dimensional measurements and to report the first observations of bump-on-tail reduced distributions at the foreshock boundary. A later publication will present more observations in a morphological study of the electron foreshock.

Observational Result

The data presented here were obtained from the Coddard Vector Electron Spectrometer experiment on ISEE-1 [Ogilvie et al., 1978]. The instrument is fully three dinensional and consists of six detectors, each with a narrow field of view ( 8.5 degrees $x 11$ degrees), which sampie the energy range 7-500 $\mathrm{eV}$ in 16 approximately logarithmically spaced energy steps in 0.5 second. The energy cycle is repeated six times per spacecraft rotation and constitutes the basic measurement which is repeated each 9 seconds. In one three second rotation period, 576 vector measurements of the velocity distribution $f\left(v_{x}, v_{y}, v_{z}, t\right)$ are made. The data are transformed to the cylindrical coordinate system which is symmetric about the measured magnetic field vector, $\underline{B}$, and which is at rest in the frame moving with the electron bulk velocity. This reduces the four-space $\left(f, v_{x}, v_{y}, v_{z}\right)$ to three dimensions $\left(f, w_{1}, w_{1}\right)$, where $w_{1}$ and $w_{n}$ are the electron proper frame velocities perpendicular and parallel to $\underline{B}$, respectively. In this way it is possible to compute the reduced distribution, $F\left(w_{n}\right)$, from measurements of the three-dimensional distribution using

$$
F\left(w_{n}\right)=2 \pi \int f\left(w_{1}, w_{n}\right) w_{1} d w_{1} .
$$

An example of an electron velocity distribution measured just inside the leading edge of the foreshock is shown in Figure 2 (upper panel) and the reduced distribution computed from it using Equation 1 is shown in Figure 2 (center panel). The integration of $w_{1} f\left(w_{1}, w_{11}\right)$ was performed in strips at constant $w_{n}$ spaced $\Delta w_{n}=5 \times 10^{7} \mathrm{~cm} / \mathrm{s}$ apart. The integration $1 \mathrm{~lm}$ ts of $w_{1}$ were 
extended by a smooth extrapolation to $w_{1} f=0$ as $w_{1} \rightarrow \infty$. The magnetic field points to the right in Figure 2, which as shown in the sketch of the geometry for this event in Figure 1, was directed toward the point of tangency from the spacecraft. Therefore, electrons moving away from the shock along the connected field line will have negative values of $w_{n}$ on the plot. We see in this example that there is a peak in the contour map and a bump on the tall of $F\left(w_{n}\right)$ at $w_{n}=-7 \times 10^{8} \mathrm{~cm} / \mathrm{s}$. There are also closed contours at $w_{n}=+7 \times 10^{8}$ $\mathrm{cm} / \mathrm{s}$ which outline a local minimum in the $f\left(w_{1}, w_{11}\right)$ surface. There is a plateau in $F\left(w_{n}\right)$ corresponding to this minimum which appears to mirror the bump on tall. This presence of a return flux of electrons toward the shock is not an uncommon feature at the foreshock boundary.

Before interpreting the contours further, we briefly review the analysis method by which they are produced. The underlying points are the center coordinates of 12 degree pltch angle-speed bins into which measurements made during one spin have been sorted, compatible with the field of view of the detectors. The surface of $f\left(w_{1}, w_{n}\right)$ is represented in three dimensions by a mesh of interconnecting triangles, formed by clusters of three nearest neighboring data points. The $f\left(w_{1}, w_{n}\right)$ surface in the space between measured data points is linearly interpolated using the three data points at the triangle vertices that bound the given region. The exception to this approach is in the cone-like data vold about the $w_{11}$-axis. This region is filled with data by an interpolating spline that is computed over pitch angle on constant speed shells, followed by triangulation of the interpolated points in til s sme way as the other data. Because of the spline interpolation, the representation of the $f\left(w_{1}, w_{n}\right)$ surface along the $w_{n}$-axis will depend on 1) the plisi angle gradients near the edge of the data void, and on 2) the assumed cylindrical symmetry about the $w_{1}$-axis. If there are weak pitch angle gradients, then the contours will tend to be circular as is the case for speeds below $3 \times 10^{8} \mathrm{~cm} / \mathrm{s}$. On the other hand, the peak in the contour map on the negative $w_{n}$-axis is the result of a well-developed trend in the measured data and the assumed symmetry. The smooth variation in the data near the edge of the data vold extends over a large enough phase space element to include measurements in several 0.5 second energy sampling cycles, which suggests that these data are not seriously time-aliased. 
Although the peak in this $f\left(w_{-}, w_{11}\right)$ contour map lies on the negative $w_{n}$-axis in a region sampled only by interpolated data, the relevant velocity space producing the bump on the $F\left(w_{n}\right)$ distribution lies off the axis and includes a range of $w_{2}$ sampled by actual measurements. We show this in the bottom panel of Figure 2 in which iso-contours of the integrand of Equation 1 , $w_{1} f\left(w_{1}, w_{n} *\right)$, normalized for each $w_{n}^{*}$ to the maximum, $\left(w_{1} f\left(w_{1}, w_{1} *\right)\right)_{\text {max }}$, are plotted on the $w_{1}-w_{n}$ grid. Because of the $w_{1}$ weighting factor, the integrand has a maximum off the $w_{1}=0$ axis (contour level 1.0) and decreases with respect to $w_{1}$ on either side of the maximum (contour levels $0.8,0.6,0.4$, and 0.2). The contour separation broadens and extends well into the velocity space sampled by actual measurements in the parallel velocity strip producing the positive slope of the bump on $F\left(w_{11}\right)$ (highlighted by the two vertical dashed lines in Figure 2). Note also that there is a sharply defined parallel velocity at which this broadening occurs. The nearly vertical alignment of the contours means that there is a lower limit to the parallel velocity of the upstream electrons, $w_{c}$, which is a confirmation of the time of filght mechanısm. Compare this feature with the more gradual spreading of the contours in the right half plane in which the solar wind heat flux electrons have no sharply defined lower Imit in $w_{n}$.

A second example of a bump on the reduced distribution is shown in Figure 3. This is a case in which there was excellent data coverage close to the magnetic field direction. The elongated, elevated contour at the parallel velocity, $w_{b}$, in the $f\left(w_{1}, w_{n}\right)$ map and the $w_{1} f$ contour map in the bottom panel clearly show that the perturbations to the distribution function have a wide range in $w_{1}$ and a lower bound, $w_{c}$, in $w_{11}$. This organization of upstream velocities according to $w_{n}$ is a basic prediction of the time of flight model sketchet in Figure 1.

The vertical bars on the $F\left(w_{11}\right)$ plots in Figure 2 and Figure 3 are the 68\% confidence intervals of the reduced distribution. The propagation of errors in determining $F\left(w_{11}\right)$ is complicated by the interdependence of neighboring elements of the $f\left(w_{1}, w_{11}\right)$ surface and oy the fact that $F\left(w_{11}\right)$ is an integral over that surface (Equation 1). Therefore, a Monte Carlo technique has bes.l used wnich takes into account the propagation of both systematic ald statistical errors. The expected uncertainties in electron counts based on 
Poisson statistics were added to the data, the $f\left(w_{1}, w_{n}\right)$ surface was determined from the noise-added data, and the reduced distribution was computed. This process was rejeated until the spectrum of nolse fluctuations in $F\left(w_{n}\right)$ no longer changed. With each computation of $F\left(w_{n}\right)$, the distribution was tested for a measurably significant positive slope. A total of 25 independent reduced distributions were computed from the noise-added data, and the bump on tall in Figure 2 and Figure 3 was present in 23 and 17 cases, respectively. We conclude that these are statistically significant bump-on-tail distributions. Furthermore, these reduced distributions have been found to be unstable according to the Penrose criterion [e.g., as in Krall and Trivelpiece (1973)].

\section{Discussion}

The magnetic field configuration and spacecraft location were particularly well suited for observing the foreshock boundary on November 6, 1977, the day of the measurements shown here. The geometry of the event shown in Figure 1 remained farly steady for several hours on that day, between 1000-1600 UT, approximately. With the leading edge of the foreshock near the spacecraft, short time scale changes in magnetic field direction caused the boundary pattern to sweep back and forth past the spacecraft many tumes during this period. Approximately 165 reduced distributions with a potential bump-on-tail were selected and 60 had measurable positive slopes that were significant to a 68\% level of confidence or better based on the error analysis outlined in the previous section. The remaining cases were rejected as resolved bump-on-tail distributions because the phase space sampling of the measurement and counting rate conspired to affect the reliability of the $F\left(w_{11}\right)$ calculation near the bump of the distribution.

The geometry of the boundary sketched in Figure 1 predicts that the distance between different parallel velocity characteristics increases with impact distance along the connecting fleld line to the shock and, therefore, the boundary in these clrcumstances should become more resolvable. During the time of these observations, the impact distance increased from 1 Re to 10 Re, and the number of cases in which two or more consecutive three second measurements 9 seconds apart in a given boundary crossing did in fact 
increase. There was also a corresponding increase in consecutive measurements of bump-on-tail reduced distributions as the impact distance increased and a significant fraction of the total (22 of 60 cases) occurred in groups of two or more. This strongly suggests that the peaked reduced distributions are a persistent feature of the foreshock boundary.

Conclusion

The persistence of Penrose unstable beams on the reduced distribution function for macroscopic times $(\Delta t=3-15 \mathrm{sec})>300 \mathrm{w}^{-1}$ is now an established fact at the electron foreshock. The kinetic explanation for the persistence of such beams remains for future theoretical work, since the simple oscillating two stream (OTS) scenario discussed by Filbert and Kellogg [1974] as adapted from Papadopoulos et al., [1974] does not explain the stabilization of the beam at such low energles. Recent, more general OTS schemes [Freund et al., 1981] of beam stabllization in the presence of suprathermal background solar wind electrons may prove to be more relevant to the actual circunstances of the foreshock. If this can be shown, ion-acoustic noise should be found in the locales of persistent beams. These and/or other explicit considerations of the inhomogeneous, driven character of the foreshock regime may lead to a kinetic explanation of the morphology presented above.

Acknowledgments. We thank Richard Thompson for a computer routine which we used in transforming measurements of the velocity distributions onto a rectangular grid suitable for contouring. 


\section{References}

Anderson, K. A., R. P. Lin, F. Martel, C. S. Lin, G. K. Parks, and H. Reme, Thin sheets of energetic electrons upstream from the earth's bowshock, Geophys. Res. Lett., 6, 401, 1979.

Anderson, R. R., G. K. Parks, T. E. Eastman, D. A. Gurnett, and L. A. Frank, Plasma waves associated with energetic particles streaming into the solar wind from the earth's bowshock, J. Geophys. Res., 86, 4493, 1981.

Feldman, W. C., J. R. Asbridge, S. J. Bame, and M. D. Montgomery, Solar wind heat transport in the vicinity of the earth's bowshock, J. Geophys. Res.. 78, 3697, 1973.

Feldman, W. C., R. C. Anderson, J. R. Asbridge, S. J. Bame, J. T. Gosling, and R. D. Zw1ckl, Plasma electron signature of magnetic connection to the earth's bowshock: ISEE-3, J. Geophys. Res., 87, 632, 1982.

Feldman, W. C., R. C. Anderson, S. J. Bame, S. P. Gary, J. T. Gosling, D. J. McComas, M. F. Thomsen, G. Paschmann, and M. M. Hoppe, Electron velocity distributions near the earth's bowshock, J. Geophys. Res., 88, 96, 1983.

Filbert, P. C. and P. J. Kellogg, Electrostatic noise at the plasma frequency beyond the earth's bowshock, J. Geophys. Res., 84, 1369, 1979.

Freund, H. P., R. A. Smith, and K. Papadopoulos, Modulational Instability in a plasma with a suprathermal tall, Phys. Of Fluids, 24, 442, 1981.

Fung, S. F., K. Papadopoulos, and C. S. Wu, Generation of electron plasma waves in the upstream solar wind, J. Geophys. Res., 87, 8077, 1982.

Greenstadt, E. W., Phenomenology of the earth's bowshock system. A summary description of experimental results, in Magnetospheric Particles and Fields, edited by B. M. McCormac, D. Reidel, Hingham, MA, 1976.

Gurnett, D. A. and L. A. Frank, Electron plasma oscillations associated with type III radio emissions and solar electrons, Solar Phys., 45, 477, 1975.

Krall, N. A. and A. W. Trivelpiece, Principles of Plasma Physics, McGraw Hill, New York, 1973.

Oglivie, K. W., J. D. Scudder, and M. Suglura, Electron energy flux in the solar wind, J. Geophys. Res., 76, 8165, 1971.

Ogluvie, K. W., J. D. Scudder, and H. Doong, The electron spectrometer experiment on ISEE-1, IEEE Irans. Geosc1. Electron., GE-16, 221, 1978.

Papadopoulos, K., M. L. Coldstein, R. A. Smith, Stabilization of electron streams in type III solar rad10 bursts, Astrophys. J., 190, 175, 1974. 
Scarf, F. L., R. W. Fredericks, L. A. Frank, and M. Neugebauer, Non-thermal electrons and high-frequency waves in the upstream solar wind. 1. Observations, J. Geophys. Res., 76, 5162, 1971. 


\section{Figure Captions}

F1g. 1. Sketch of electron foreshock boundary showing the spatial dispersion with respect to parallel velocity which generates the bump-on- tail reduced distributions.

Fig. 2. Contour map of the surface $f\left(w_{2}, w_{n}\right)$ (upper panel) obtained from a three-dimensional measurement of the electron velocity distribution at the electron foreshock boundary on November 6 , 1977 at 15:39:41-44 UT. The points underlying the contours locate the measurements in velocity space. The reduced distribution, $F\left(w_{n}\right)$, (center panel) shows a bump on the tail at $w_{n}=-7 \times 10^{8} \mathrm{~cm} / \mathrm{s}$, and the vertical dashed lines outline the elemental strip of parallel velocity space producing the positive slope. The enhancement in $F$ at $w_{1}=+7 \times 10^{8} \mathrm{~cm} / \mathrm{s}$, on the other hand, does not have a positive slope that can be supported by the propagation of errors analysis discussed in the text. (Lower panel) Contour map of $w_{1} f\left(w_{1}, w_{11}\right)$ (normalized) showng the relative contribution of perpendicular velocities to $F\left(w_{n}\right)$ at each parallel velocity.

F1g. 3. Another example of the bump-on-tail reduced distributions measured at the foreshock boundary on November 6, 1977 at 09:54:04-07 UT. The points underlying the contours locate the measurements in velocity space and show that there was good data coverage along the magnetic field direction. 


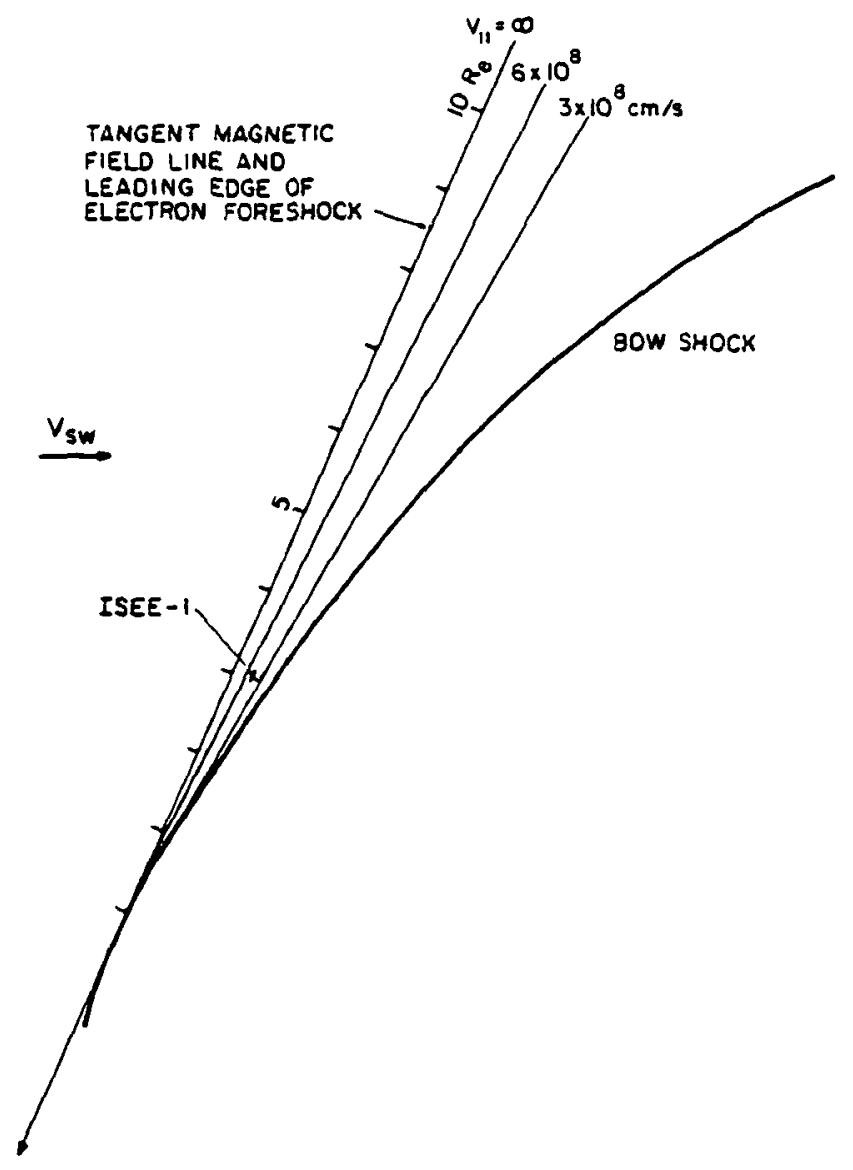

Figure 1 


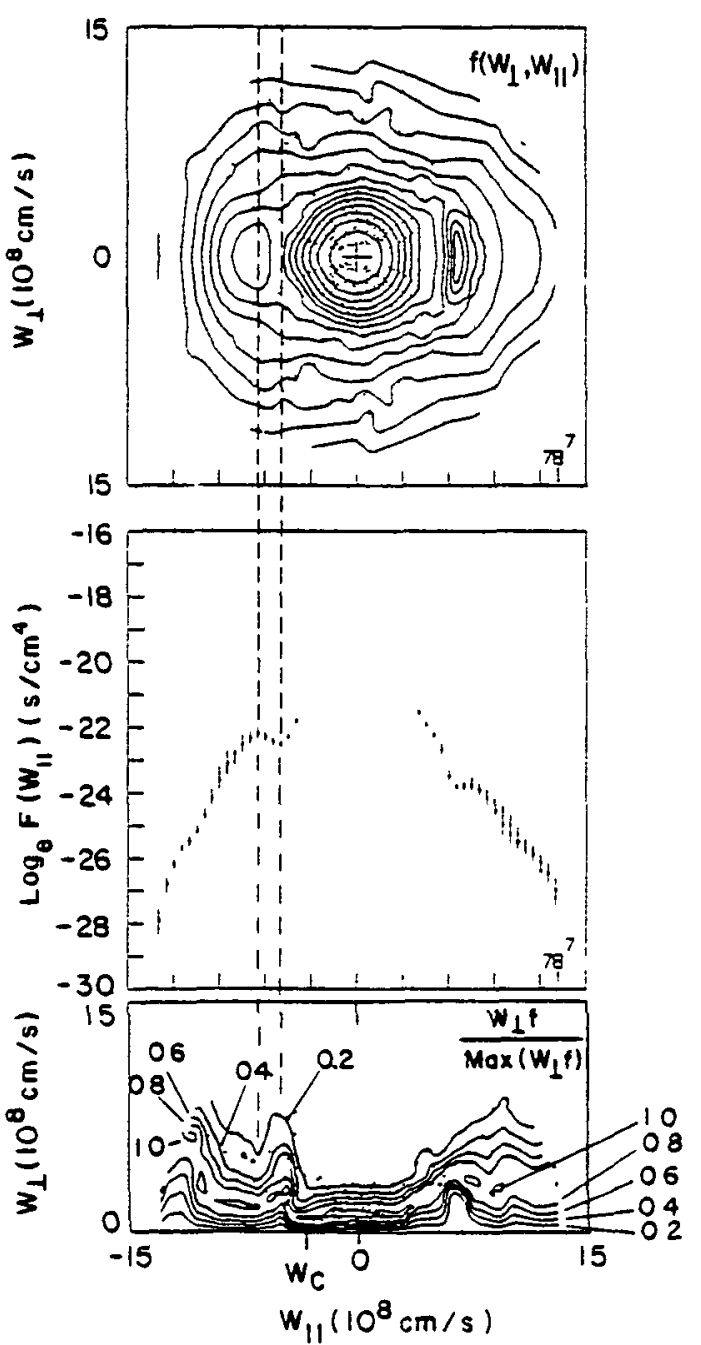

Figure 2 


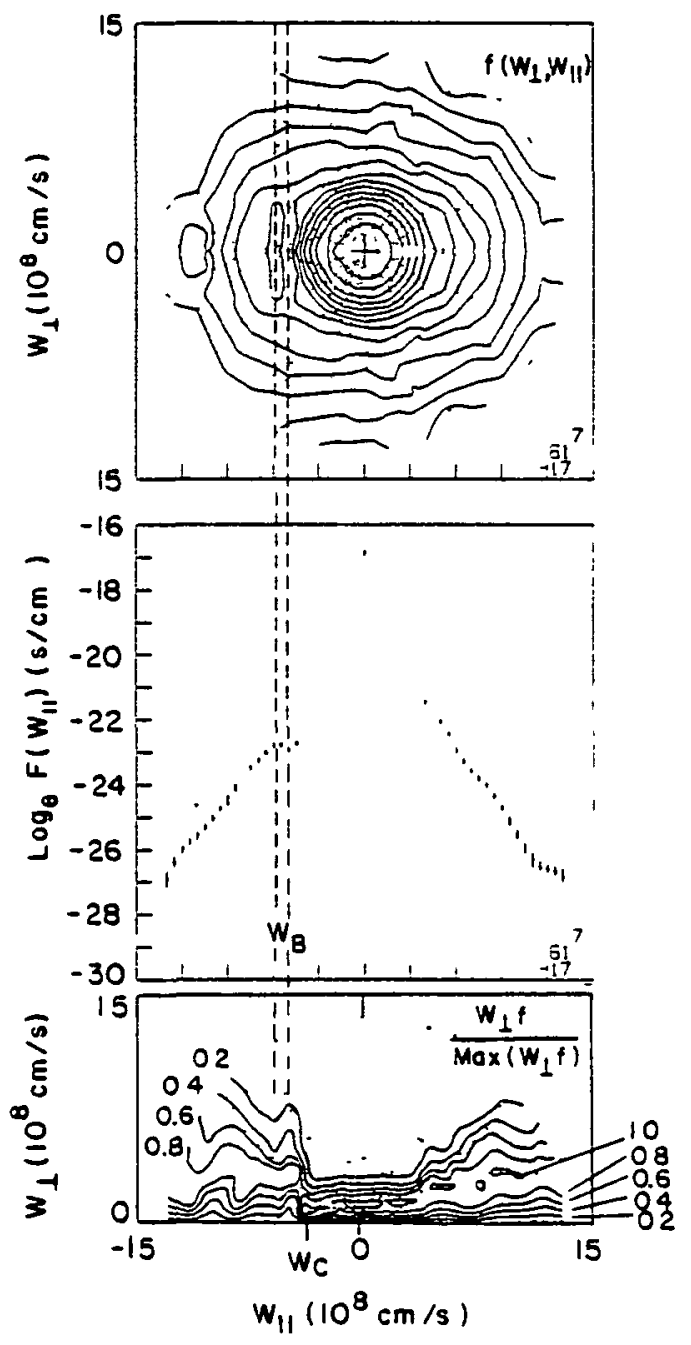

Figure 3 
BIBLIOGRAPHIC DATA SHEET

\begin{tabular}{|c|c|}
\hline 2. Government Accession No. & 3. Recipient's Catalog No. \\
\hline \multirow{2}{*}{$\begin{array}{l}4 \text { Title and Subtitle } \\
\text { Detection of Bump-on-Tail Reduced Electron } \\
\text { Velocity Distributions at the Electron Foreshock } \\
\text { Boundary }\end{array}$} & $\begin{array}{l}5 \text { Report Date } \\
\text { February } 1984\end{array}$ \\
\hline & 6. Performing Organızation Code \\
\hline $\begin{array}{l}\text { 7. Author(s) Richard J. Fitzenreiter, Alexander J. } \\
\text { KI imas and Jack D. Scudder }\end{array}$ & 8. Performing Organization Report No. \\
\hline \multirow{3}{*}{$\begin{array}{l}\text { 9. Performing Organization Name and Address } \\
\text { NASA/GSC } \\
\text { Laboratory for Extraterrestrial Physics } \\
\text { Interplanetary Physics Branch, Code } 692 \\
\text { Greenbelt, MD } 20771\end{array}$} & 10. Work Unit No. \\
\hline & 11. Contract or Grant No. \\
\hline & 13. Type of Report and Period Covered \\
\hline \multirow[t]{2}{*}{ 12. Sponsoring Agency Name and Address } & Technical Memorandum \\
\hline & 14. Sponsoring Agency Code \\
\hline
\end{tabular}

15. Supplementary Notes

16. Abstract Reduced velocity distributions are derived from three-dimensional measurements of the velocity distribution of electrons in the 7-500 eV range in the electron foreshock. Bump-on-tail reduced distributions are presented for the first time at the foreshock boundary consistent with Filbert and Kellogg's proposed time-of-flight mechanism for generating the electron beams. In a significant number of boundary crossings, bump-on-tail reduced distributions were found in consecutive $3 \mathrm{sec}$ measurements made $9 \mathrm{sec}$ apart. We conclude that, although the beams are linearly unstable to plasma waves according to the Penrose criterion, they persist on a time scale of $3-15 \mathrm{sec}$.

17. Key Words (Selected by Author(s)) Solar wind plasma, plasma instabilities, interactions between solar wind and magnetosphere

18. Distribution Statement

\begin{tabular}{|c|c|c|c|}
\hline $\begin{array}{l}\text { 19. Security Classif lof this report) } \\
U\end{array}$ & $\begin{array}{l}\text { 20. Security Classif (of this page) } \\
\text { U }\end{array}$ & $\begin{array}{l}\text { 21. No. of Pages } \\
15\end{array}$ & 22. Price \\
\hline
\end{tabular}

